\title{
In-Situ Observation of Concurrent Oxidation and Mechanical Deformation in Al and $\mathbf{Z r}$
}

Yang Yang ${ }^{1,2}$, Akihiro Kushima ${ }^{3}$, Huolin Xin ${ }^{4 *}$, Peter Hosemann ${ }^{5}, \mathrm{Ju} \mathrm{Li}^{1 *}$

1. Nuclear Science and Engineering, Massachusetts Institute of Technology, Cambridge, USA.

2. National Center for Electron Microscopy, Molecular Foundry, Lawrence Berkeley National Laboratory, Berkeley, USA.

3. Department of Materials Science and Engineering, University of Central Florida, Orlando, USA

4. Department of Physics and Astronomy, University of California, Irvine, USA

5. Department of Nuclear Engineering, University of California, Berkeley, USA

*Corresponding author: huolinx@uci.edu, liju@mit.edu

Metals will naturally form an oxide layer at the surface when exposed to air. The oxide skin is a diffusion barrier that may slow down the corrosion rate significantly. Since structural materials are usually working under external/internal stresses, cracks may be generated inside the oxide layer during deformation, reducing the protectiveness of oxide. Understanding the response of metal surface oxide to concurrent chemical reaction and deformation, is critical for unravelling the stress corrosion cracking (SCC) mechanisms of metals and development of alloys with improved corrosion-resistance. However, direct observation of the physical processes in the oxide layer as well as at the metal-oxide-interface (MOI) is challenging, as they are usually buried by nature [1]. Herein, using an environmental TEM (ETEM), we report in-situ observations of the concurrent oxidation and mechanical deformation in aluminum (Al) and zirconium ( $\mathrm{Zr}$ ). These two metals are interesting systems to compare: $\mathrm{Al}$ has an amorphous native oxide at room temperature while $\mathrm{Zr}$ develops a crystalline oxide.

For Al, we develop a mechanical break-junction approach enabling direct observation of the environmental deformation of the naturally-formed alumina on Al surface at room temperature. The experimental procedures are illustrated in Fig. 1a-c. We prepare two Al nanotips, and bring them to contact inside an ETEM (Fig. 1a). The nanotips are cold-welded [2] together (Fig. 1b). Afterwards, pure oxygen is injected inside the chamber (Fig.1c). We then pull the nanotips and observe the evolution of the Al oxide layer and MOI simultaneously. We found that native alumina is liquid-like during deformation, and it can remain its integrity without any cracks/spallation (Fig. 1d-e) at moderate strain rate. Also, it can keep matching the deformation of metal even when the metal surface inclinations have changed significantly. When the strain rate is higher, fracture in metal will occur, exposing fresh metal surface. We then visualized the initial oxidation of $\mathrm{Al}$ at the metal-gas interface at atomic resolution (Fig. 2a-c). Unlike traditional thin film growth or nanoglass consolidation processes, we observe seamless coalescence of new oxide islands without forming any glass-glass interface or surface grooves (Fig. 2b), indicating greatly accelerated glass kinetics [3] at the surface compared to the bulk.

For $\mathrm{Zr}$, we visualize the high temperature oxidation process when no external force is applied. It is surprising that the oxidation-induced internal stress lead to direct cracks/pores formation in the thin film (fig. 2d), which in turn accelerates the oxidation kinetics by providing new oxygen diffusion shortcuts.

In conclusion, our research reveals the liquid-like superplasticity in thin amorphous alumina during deformation at room temperature. Our findings have important implications for not only understanding of the unique oxidation resistance in $\mathrm{Al}$, but also development of new corrosion-resistant coatings and alloys. 


\section{References:}

[1] L. Zou, J. Li, D. Zakharov, E. A. Stach, and G. Zhou, Nat. Commun. 8, 307 (2017).

[2] Y. Lu, J. Y. Huang, C. Wang, S. Sun, and J. Lou, Nat. Nanotechnol. 5, 218 (2010).

[3] F. G. Sen, A. T. Alpas, A. C. T. van Duin, and Y. Qi, Nat. Commun. 5, 1 (2014).

[4] Y. Yang, A. Kushima, W. Han, H. L. Xin, and J. Li, Nano Lett. (2018).
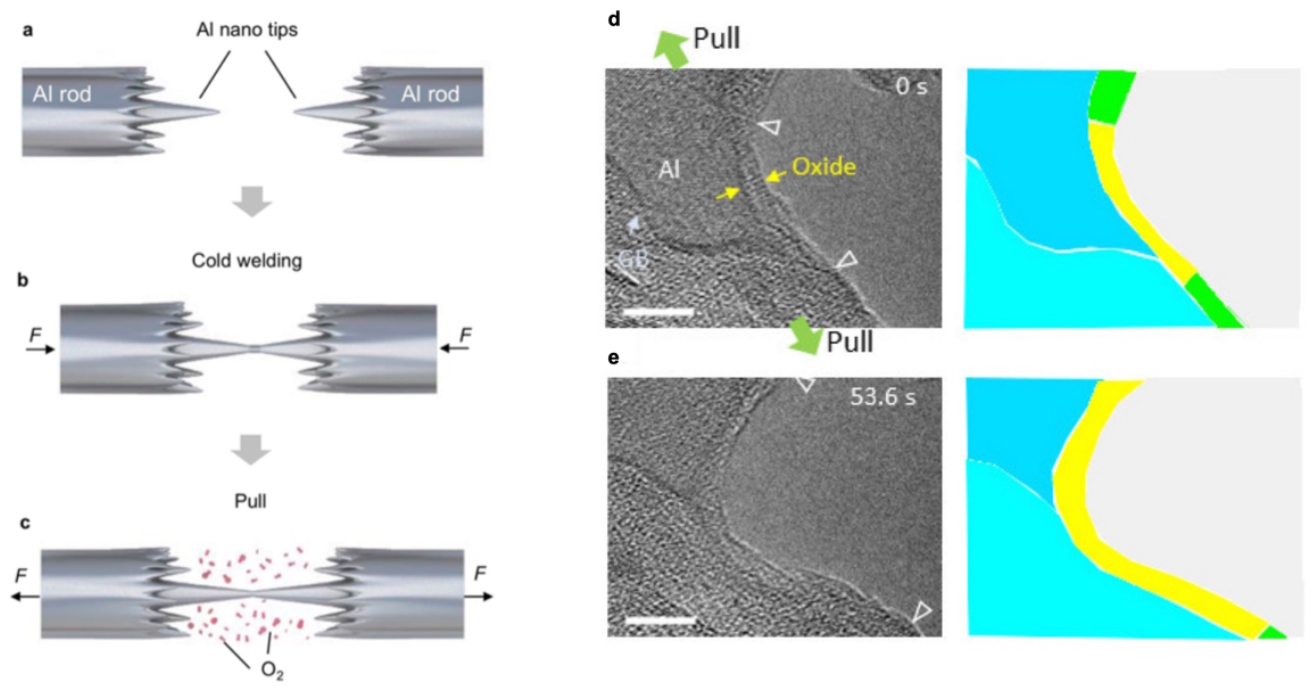

Al grain 1 (on zone axis)

Al grain 2 (off zone axis)

Oxide (under tension)

Oxide (not stressed)

Oxygen gas

Figure 1. $(\mathrm{a}-\mathrm{c})$ Schematic drawing of the experimental procedures. (d-e) Sequential TEM images showing the super-elongation and self-healing process of aluminum oxide when stretched in $\mathrm{O}_{2}$ environment. The strain of oxide at (e) is about 117\%. All scale bars, $5 \mathrm{~nm}$. Reprinted with permission from [4]. Copyright 2018 American Chemical Society.

a
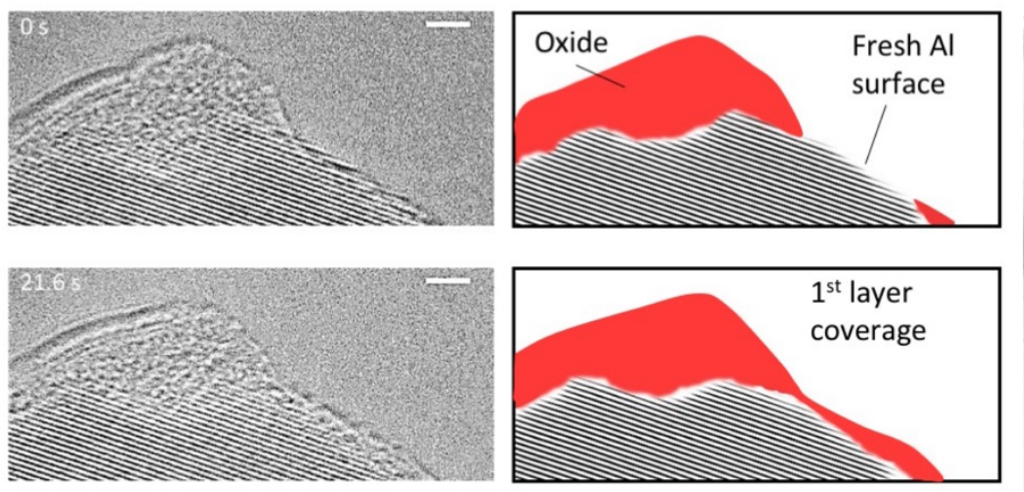

c
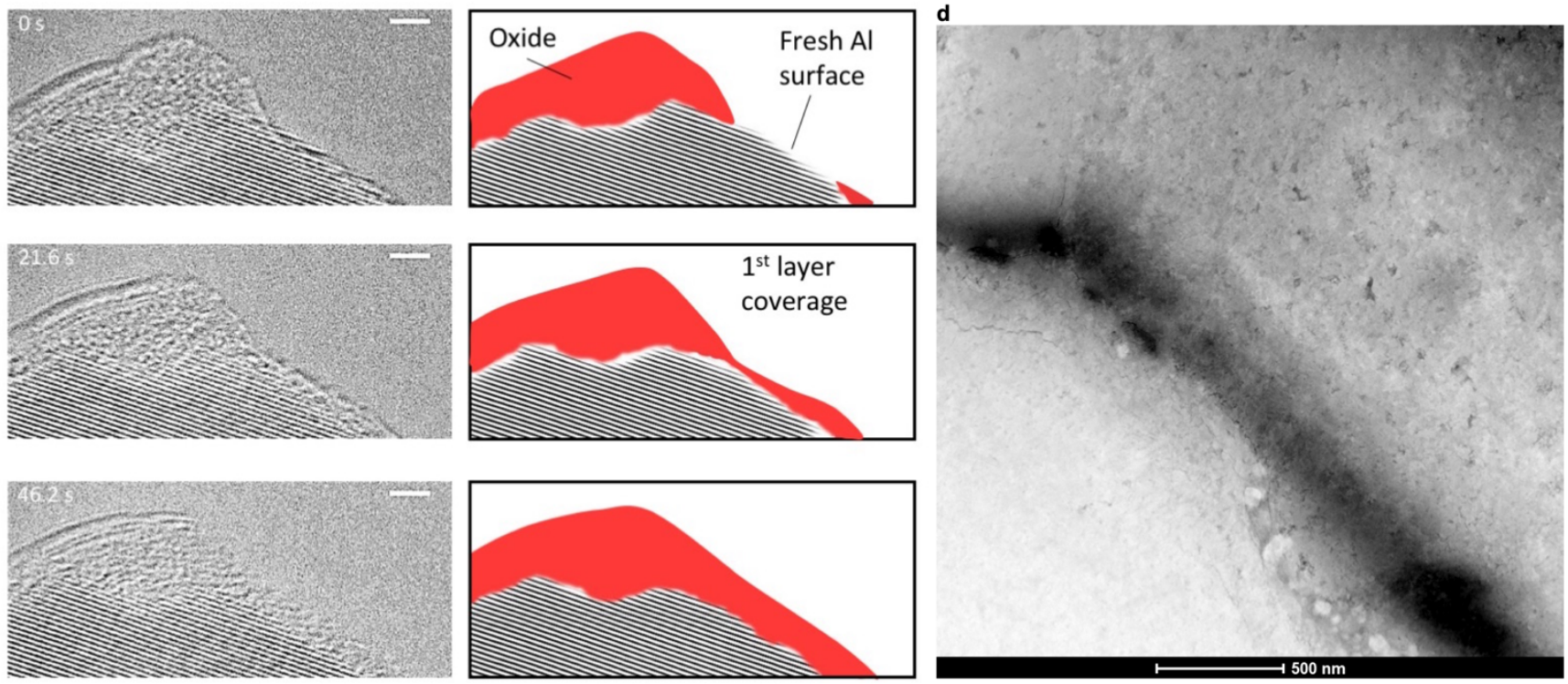

Figure 2. (a-c) Sequential HRTEM images of the initial oxidation process on aluminum fresh surface. Reprinted with permission from [4]. Copyright 2018 American Chemical Society. (d) HAADF image of the $\mathrm{Zr}$ thin film after high temperature oxidation, showing numerous small cracks and pores. 